\title{
"Subclinical" pacemaker syndrome: a randomised study of symptom free patients with ventricular demand (VVI) pacemakers upgraded to dual chamber devices
}

Department of Cardiology, Guy's Hospital, London N Sulke

A Dritsas

J Bostock

A Wells

$\mathrm{R}$ Morris

Correspondence to Dr Neil Sulke,

Department of Cardiology, Guy's Hospital, St Thomas' Street, London SE1 9RT.

Accepted for publication 12 September 1991

\begin{abstract}
Objective-To determine whether symptom free patients with single chamber pacemakers benefit from dual chamber pacing.

Design-A randomised double blind crossover comparison of ventricular demand (VVI), dual chamber demand (DDI), and dual chamber universal (DDD) modes after upgrading from a VVI device.
\end{abstract}

Setting-Cardiology outpatient department.

Patients-Sixteen patients aged 41-84 years who were symptom free during VVI mode pacing for three or more years.

Intervention-Pacemaker upgrade during routine generator change.

Main outcome measures-Change in subjective (general health perception, symptoms) and objective (clinical assessment, treadmill exercise, and radiological and echocardiographic indices) results between pacing modes before and after upgrading.

Results-75\% preferred DDD, $68 \%$ found VVI least acceptable with $12 \%$ expressing no preference. Perceived general well-being and exercise capacity $(\mathbf{p}<0.01)$ and treadmill times $(p<0.05)$ were improved in DDD mode but VVI and DDI modes were similar. Clinical, echocardiographic, radiological, and electrophysiological indices confirmed the absence of overt pacemaker syndrome, although mitral and tricuspid regurgitation was greatest in VVI mode $(\mathbf{p}<0.01)$.

Conclusions-Most patients who were satisfied with long term pacing in VVI mode benefited from upgrading to DDD mode pacing suggesting the existence of "subclinical" pacemaker syndrome in up to $75 \%$ of such patients. The DDI mode offes ed little subjective or objective benefit over VVI mode in this population and should be reserved for patients with paroxysmal atrial arrhythmias.

VVI mode pacing should be used only for patients with very intermittent symptomatic bradycardia or atrial fibrillation with a good chronotropic response during exercise.
The pacemaker syndrome is a constellation of signs and symptoms arising as a direct result of ventricular demand pacing (VVI mode). ${ }^{1}$ The mechanisms of this syndrome are varied and may be the result of haemodynamic, neuroendocrine, and electrophysiological factors. $^{2-4}$

In most cases this syndrome can be cured by dual chamber pacing, which relieves almost all the symptoms. ${ }^{5}$ However, there are several reports of "pacemaker syndrome" occurring in dual chamber modes ${ }^{6} 7$ (usually explained by adverse programming of atrioventricular delay causing, in effect, ventricular asynchronous pacing).

The incidence of the pacemaker syndrome in VVI paced patients (still the predominant pacing mode used worldwide and in the United Kingdom) has been variously described as $7-25 \%$ according to the severity of intolerance of ventricular asynchronous pacing. ${ }^{8}$ No study has yet investigated the effect of upgrading VVI devices to dual chamber pacing in apparently symptom free patients.

\section{STUDY AIMS}

To assess, by a randomised double blind crossover study the effect of dual chamber pacing in patients with no overt evidence of the pacemaker syndrome after chronic, longterm VVI pacing.

\section{Patients and methods}

PATIENTS

We studied 16 patients aged 41-84 years (mean 66.6 years, $69 \%$ male). Any patient who was largely symptom free and who felt "generally well" after chronic VVI pacing for at least three years could be enrolled. Table 1 shows the reasons why patients needed a change of pacemaker generator. Patients with chronic atrial flutter or fibrillation, or who were unable to walk on a treadmill were excluded. One patient with a VVIR pacemaker programmed to VVI mode for three years was included.

STUDY DESIGN

This was a prospective randomised double blind crossover comparison of three pacing modes (VVI, DDI, and DDD) after a VVI pacemaker had been upgraded to a dual chamber device in patients expressing no dis- 
Table 1 Patient details, pacemaker types, and left ventricular systolic function

\begin{tabular}{|c|c|c|c|c|c|c|c|c|c|c|c|c|c|}
\hline Patient & Sex & Age & $\begin{array}{l}\text { Indication } \\
\text { for } \\
\text { implant }\end{array}$ & $\begin{array}{l}\text { Other } \\
\text { associated } \\
\text { conditions }\end{array}$ & $\begin{array}{l}\text { Pacemaker } \\
\text { dependent }\end{array}$ & $\begin{array}{l}\text { Date of } \\
\text { initial } \\
\text { implant }\end{array}$ & $\begin{array}{l}\text { Previous } \\
\text { pacemaker } \\
\text { type }\end{array}$ & $\begin{array}{l}\text { Reason } \\
\text { for } \\
\text { upgrade }\end{array}$ & $\begin{array}{l}\text { Upgrade } \\
\text { pacemaker } \\
\text { type }\end{array}$ & $\begin{array}{l}\text { Pacing } \\
\text { base } \\
\text { rate }\end{array}$ & $\begin{array}{l}\text { Peak } \\
H R \text { in } \\
V V I\end{array}$ & $\begin{array}{l}\text { Peak } \\
H R \text { in } \\
D D D\end{array}$ & $\begin{array}{l}\text { Mean } L V \\
\text { fractional } \\
\text { shortening }(\%\end{array}$ \\
\hline $\begin{array}{l}1 \\
2\end{array}$ & $\begin{array}{l}\mathbf{M} \\
\mathbf{M}\end{array}$ & $\begin{array}{l}69 \\
74\end{array}$ & \multirow{2}{*}{$\begin{array}{l}\text { SSS + AVIII } \\
\text { Syncope } \\
\text { AVI, AVII } \\
\text { AVIII }\end{array}$} & MI, PAF & $\begin{array}{l}\mathbf{Y} \\
\mathbf{N}\end{array}$ & $\begin{array}{l}1980 \\
1979\end{array}$ & $\begin{array}{l}\text { S } 625^{\star} \\
\text { ELA } 1250\end{array}$ & $\begin{array}{l}\text { EOL } \\
\text { EOL }\end{array}$ & $\begin{array}{l}\text { Delta } \\
\text { Cosmos II }\end{array}$ & $\begin{array}{l}55 \\
70\end{array}$ & $\begin{array}{r}55 \\
165\end{array}$ & $\begin{array}{l}115 \\
140\end{array}$ & $\begin{array}{l}12 \\
42\end{array}$ \\
\hline 3 & $\mathbf{M}$ & 75 & & \multirow[t]{3}{*}{$\begin{array}{l}\text { DM, TIAs } \\
\text { MI }\end{array}$} & $\mathbf{Y}$ & 1979 & S 668 & EOL & Cosmos II & 70 & 70 & 88 & 13 \\
\hline 4 & $\mathbf{F}$ & 67 & \multirow{2}{*}{$\begin{array}{l}\text { Intermittent } \\
\text { AVII, AVIII } \\
\text { AVIII }\end{array}$} & & $\mathbf{N}$ & 1977 & TE 120B & EOL & Cosmos II & 70 & 126 & 120 & 40 \\
\hline 5 & $\mathbf{M}$ & 71 & & & $\mathbf{Y}$ & 1982 & APC 5251 & $\begin{array}{l}\text { Faulty reed } \\
\text { switch }\end{array}$ & Cosmos II & 70 & 70 & 87 & 20 \\
\hline 6 & $\mathbf{M}$ & 41 & AVIII & \multirow[t]{2}{*}{$\begin{array}{l}\text { Dilated } \\
\text { cardio- } \\
\text { myopathy }\end{array}$} & $\mathbf{Y}$ & 1976 & S 668 & EOL & AFP & 75 & 80 & 145 & 14 \\
\hline $\begin{array}{l}7 \\
8 \\
9\end{array}$ & $\begin{array}{l}M \\
M \\
F\end{array}$ & $\begin{array}{l}72 \\
77 \\
65\end{array}$ & $\begin{array}{l}\text { AVIII } \\
\text { AVIII } \\
\text { AVIII }\end{array}$ & & $\begin{array}{l}\mathbf{Y} \\
\mathbf{Y} \\
\mathbf{Y}\end{array}$ & $\begin{array}{l}1978 \\
1977 \\
1985\end{array}$ & $\begin{array}{l}\text { C L900 } \\
\text { TE } 120 \mathrm{~B} \\
\text { TE } 5281\end{array}$ & $\begin{array}{l}\text { EOL } \\
\text { EOL } \\
\text { Painful } \\
\text { pacemaker } \\
\text { site }\end{array}$ & $\begin{array}{l}\text { Cosmos II } \\
\text { Cosmos II } \\
\text { AFP }\end{array}$ & $\begin{array}{l}70 \\
70 \\
70\end{array}$ & $\begin{array}{l}70 \\
70 \\
70\end{array}$ & $\begin{array}{l}109 \\
130 \\
140\end{array}$ & $\begin{array}{l}23 \\
21 \\
36\end{array}$ \\
\hline $\begin{array}{l}10 \\
11\end{array}$ & $\underset{\mathrm{M}}{\mathrm{F}}$ & $\begin{array}{l}57 \\
65\end{array}$ & $\begin{array}{l}\text { SSS + AVIII } \\
\text { SSS + AVIII }\end{array}$ & \multirow[t]{2}{*}{$\begin{array}{l}\text { PHA, PAF } \\
\text { Post MVR }\end{array}$} & $\begin{array}{l}\mathbf{Y} \\
\mathbf{Y}\end{array}$ & $\begin{array}{l}1983 \\
1982\end{array}$ & $\begin{array}{l}\text { VIT TX* } \\
\text { S } 668\end{array}$ & $\begin{array}{l}\text { EOL } \\
\text { Intermittent } \\
\text { myopotential } \\
\text { inhibition }\end{array}$ & $\begin{array}{l}\text { AFP } \\
\text { Synchrony }\end{array}$ & $\begin{array}{l}70 \\
70\end{array}$ & $\begin{array}{l}70 \\
70\end{array}$ & $\begin{array}{r}130 \\
80\end{array}$ & $\begin{array}{l}25 \\
21\end{array}$ \\
\hline $\begin{array}{l}12 \\
13 \\
14\end{array}$ & $\begin{array}{l}\mathrm{F} \\
\mathrm{F}\end{array}$ & $\begin{array}{l}74 \\
69 \\
52\end{array}$ & $\begin{array}{l}\text { SNI + AVIII } \\
\text { AVII } \\
\text { SNI, AVIII }\end{array}$ & & $\begin{array}{l}\mathbf{Y} \\
\mathbf{N} \\
\mathbf{Y}\end{array}$ & $\begin{array}{l}1978 \\
1978 \\
1987\end{array}$ & $\begin{array}{l}\text { MD } 5927 \\
\text { C L900 } \\
\text { S 688L }\end{array}$ & $\begin{array}{l}\text { EOL } \\
\text { EOL } \\
\text { Faulty } \\
\text { telemetry }\end{array}$ & $\begin{array}{l}\text { Synchrony } \\
\text { AFP } \\
\text { Synchrony }\end{array}$ & $\begin{array}{l}70 \\
70 \\
70\end{array}$ & $\begin{array}{l}70 \\
96 \\
70\end{array}$ & $\begin{array}{l}135 \\
110 \\
131\end{array}$ & $\begin{array}{l}35 \\
42 \\
25\end{array}$ \\
\hline 15 & $\mathbf{M}$ & 84 & \multirow{2}{*}{$\begin{array}{l}\text { Intermittent } \\
\text { AVIII } \\
\text { SSS + AVIII }\end{array}$} & \multicolumn{2}{|c|}{ Multiple VEsN } & 1979 & MD 5983 & EOL & Paragon & 70 & 78 & 94 & 30 \\
\hline 16 & $\mathbf{M}$ & 70 & & & $\mathbf{Y}$ & 1983 & S 678B & EOL & AFP & 70 & 70 & 125 & 32 \\
\hline
\end{tabular}

*Paced in VVI mode for more than three years before upgrading procedure.

Indication for implantation: SSS, sick sinus syndrome (including paroxysmal supraventricular tachycardia); AVI, first degree heart block; AVII, second degree heart block; AVIII, complete heart block; SNI, sinus node, chronotropic incompetence.

Other conditions: MI, myocardial infarction; PAF, paroxysmal atrial fibrillation; DM, diabetes mellitus; TIA, transient ischaemic attack; PHA, post His bundle ablation; MVR, mitral valve replacement; VE, ventricular extrasystoles.

Pacemaker dependent: Y, predominantly paced rhythm in VVI mode. N, predominately non-paced rhythm.

Reason for upgrade: EOL, end of life.

satisfaction with long term, ventricular demand pacing. Before enrolment all patients underwent a full assessment with their original pacemaker programmed to VVI mode. This was called "VVI pre" mode. If the subjective questionnaire scores suggested symptoms attributable to the "pacemaker syndrome" the patient was excluded from the study at this stage. Postoperatively all patients were programmed to the three study modes in a random sequence (by random number tables). The VVI mode programmed after upgrading was called the "VVI post" mode.

Written, informed consent was obtained from each patient before the upgrading procedure. Programming was undertaken by senior cardiac technicians and full assessment was undertaken after four weeks of out of hospital activity in each mode. After the upgrading neither the patients nor the investigating clinicians were aware of the programmed pacing mode at any time.

\section{PACEMAKER UPGRADING PROCEDURE}

The old generator was explanted and the ventricular electrode tested under local anaesthesia. If the ventricular pacing threshold was $2 \mathrm{~V}$ or less, with an intracardiac $R$ wave amplitude of $3 \mathrm{mV}$ or more the electrode was retained and adapted to fit an appropriate dual chamber device (table 1 ). If these criteria were not fulfilled a new bipolar carbon tipped electrode (Siemens-Elema model 1010T/60 (IS1), Siemens-Elema AB, Stockholm, Sweden) was inserted via the subclavian vein and the old electrode was removed. The atrial electrode was inserted via the same subclavian vein and sited in the right atrial appendage in all patients.

All but two patients (1 and 3) underwent intraoperative electrophysiological assessment. This showed normal atrioventricular nodal conduction in three patients (patients 2,4 , and 13) (atrioventricular node Wenckebach cycle length greater than 120 beats per minute); abnormal sinus node recovery time (SNRT) in five patients (patients 10,11,12, 14 and 16) (SNRT greater than $1500 \mathrm{~ms}$, corrected SNRT greater than $550 \mathrm{~ms})^{10}$; and consistent retrograde ventriculo-atrial conduction in four patients (patients 2, 4, 6 and 13).

\section{PACEMAKER PROGRAMMING}

Twenty four hours after their pacemaker was upgraded all patients were checked to ensure satisfactory dual chamber pacing and then programmed to the first randomised study mode) at a pacing basic rate identical with that in VVI pre mode. An atrioventricular delay of $150 \mathrm{~ms}$ was programmed in the DDD and DDI modes in all patients.

A settling in period of two weeks in the first mode was allowed after implantation and the first four week study period started immediately afterwards. After this study period satisfactory dual chamber pacing function and pacing thresholds were reconfirmed. The pulse amplitude in each chamber was then programmed to twice the pacing threshold and the sensitivity to half the sensing threshold. These variables remained unaltered for the rest of the study.

\section{SUBJECTIVE ASSESSMENT}

This consisted of three self administered questionnaires. The first used visual analogue scales to assess the perceived general wellbeing and exercise capacity. Subjects placed a mark on a $15 \mathrm{~cm}$ line from the discrete minimum point signifying "extremely unwell" 
or "inability to exercise" to the maximum point signifying "extremely well" or "unlimited exercise capacity". The result was expressed as a percentage of the distance from the minimum point to the mark divided by the length of the line. ${ }^{1112}$

The second questionnaire assessed the patient's perception of their functional capacity by the well validated Specific Activities Scale functional status questionnaire. ${ }^{13}$ This grades patients from class I (unlimited physical capacity) to class IV (grossly incapacitated).

The final questionnaire assessed the incidence and frequency of symptoms of mild cardiac failure or pacemaker induced haemodynamic dysfunction that occurs in the pacemaker syndrome. ${ }^{1}$ The format of the questionnaire used a quantitative score where: $1=$ all of the time, $2=$ most of the time, 3 = some of the time, $4=$ occasionally, and $5=$ none of the time. The "symptom prevalence score" (maximum 84) was calculated by the arbitrary weighting of symptoms (table 2). A score of 25 or more was highly suggestive of the pacemaker syndrome or moderate heart failure.

\section{CLINICAL ASSESSMENT}

This was undertaken in all cases by the same clinician (NS) while he was unaware of the patient's programmed mode. The clinical examination was performed after subjective and objective assessment was completed, with patients lying supine on an examination couch with their upper torso at $45^{\circ}$. Table 3 shows the

Table 2 Symptom prevalence score

\begin{tabular}{lll}
\hline & Symptom & Score \\
\hline 1 & Memory & $0-4$ \\
2 & Concentration & $0-4$ \\
3 & Tiredness & $0-8$ \\
4 & Lightheadedness & $0-8$ \\
5 & Shortness of breath & $0-12$ \\
6 & Orthopnoea & $0-12$ \\
7 & Cough & $0-4$ \\
8 & Palpitation & $0-8$ \\
9 & Fluttering in neck/abdomen & $0-8$ \\
10 & Dizziness & $0-8$ \\
11 & Ankle oedema & $0-8$ \\
Total score & $0-84$ \\
\hline
\end{tabular}

Table 3 Clinical indices assessed and the clinical assessment score

\begin{tabular}{ll}
\hline & $\begin{array}{l}\text { Clinical } \\
\text { assessment } \\
\text { score }\end{array}$ \\
\hline Variability of systolic blood pressure & $0-6$ \\
JVP cannon waves & $0-6$ \\
Peripheral oedema & $0-6$ \\
Pulmonary oedema & $0-6$ \\
Pulsatile liver & $0-2$ \\
Variability of heart sounds & $0-2$ \\
Variability of heart murmur(s) & $0-2$ \\
Characteristics of heart murmur(s) & $0-6$ \\
Effect of magnet application to pacemaker & $0-6$ \\
on characteristics of murmur(s) & $0-6$ \\
Extent of retrograde V-A conduction during & $0-6 \star$ \\
magnet application (fixed rate pacing) & $0-6{ }^{\star}$ \\
Competing atrial rhythm during normal & $0-2^{\star}$ \\
pacemaker function & $0-50$ \\
Score &
\end{tabular}

*Assessed by assisting senior cardiac technician. VP, jugular venous pulse. $\mathrm{V}$-A, ventriculoatrial. Clinical examination included evaluation of: pacemake dependency ${ }^{\star}$; concurrent medication; pulse rate, character an rhythm; blood pressure; jugular venous pulse wave level and character; myopotential inhibition ${ }^{\star}$; arrhythmia type and duration. * variables assessed and arbitrary clinical assessment score. (Any assessment requiring electrocardiographic evaluation was undertaken by the senior technician assisting in patient monitoring).

\section{OBJECTIVE ASSESSMENT}

(i) Graded exercise treadmill testing

Patients were monitored by a continuous sixlead electrocardiogram and were assessed by the Chronotropic Assessment Exercise Protocol (CAEP). ${ }^{14}$ Tests were symptom limited and the decision to stop the protocol was made by the supervising clinician alone. A senior cardiac technician monitored the electrocardiogram during all tests. Total exercise time and resting and peak heart rates were obtained for each patient.

(ii) Staircase ascent

Patients had their heart rates measured by telemetry using a Hewlett Packard 78571B ECG recorder while they climbed as many stairs as they could to a maximum of five flights (each comprising fifteen steps, $15 \mathrm{~cm}$ tall, pitch $27 \mathrm{~cm}$ ), as fast as possible. Heart rate was assessed at rest before the ascent and every 15 seconds for two minutes. The number of stairs ascended and the percentage change in heart rate were calculated.

\section{RADIOLOGICAL ASSESSMENT}

Chest radiographs were obtained 24 hours before the upgrading procedure, 24 hours postoperatively with the patient programmed to VVI mode, and finally after a four week study period programmed to a dual chamber mode (either DDI or DDD) chosen randomly by the assisting senior technician. These were designated as "VVI pre", "VVI post", and "Dual" radiographs respectively.

After completion of the study radiographs were independently analysed by two clinicians who were unaware of the patient's name, the pacing mode, or dates of the radiographs. The cardiothoracic ratio was calculated as the widest dimension of the cardiac shadow (in centimetres) divided by the widest internal dimension of the thoracic cage (in $\mathrm{cm}$ ) and expressed as a percentage. An arbitrary "pulmonary oedema severity score" was devised where: $0=$ absent, $1=$ mild, $2=$ moderate, $3=$ severe.

\section{ECHOCARDIOGRAPHIC ASSESSMENT}

A Hewlett-Packard 77020A system with a $2.5 \mathrm{MHz}$ duplex probe and a $1.9 \mathrm{MHz}$ continuous wave transducer was used. Serial studies on individual subjects were carried out by the same operator to avoid inter-observer variability.

$\mathrm{M}$-mode recordings were taken at conventional levels with dimensions estimated from leading edge to leading edge. ${ }^{15}$ To ensure that the operator remained "blinded" to the pacing mode simultaneous electrocardiographic recording was not used. This meant that the left ventricular diastolic diameter was taken as the greatest dimension in diastole and the systolic diameter as the smallest dimension in systole. 
Stroke volume was calculated as the product of the systolic velocity integral and cross sectional area for flow by a combined cross sectional Doppler technique. Subaortic crosssectional area was measured from inner to inner echo at the level of the annulus in a parasternal long axis frame frozen early in systole. ${ }^{16}$ The systolic velocity integral was calculated from the aortic envelope recorded from the apical position. This was averaged over five beats by triangulation (stroke volume index (SVI) $=$ $1 / 2 \times$ ejection time $\left.\times V_{\max }\right)$. This has been shown to be equivalent to planimetry. ${ }^{17} 18$

Pulmonary artery systolic pressure was estimated where possible from the peak velocity of the tricuspid regurgitant jet recorded by continuous wave Doppler. Pressure (p) was calculated by the formula of Berger et $a l^{19}$ where $p=6 / 5 \times 4\left(V_{\max }\right)^{2}$. Signals lacking a clearly defined envelope were discarded.

The colour flow Doppler system was set to the same enhanced threshold map for every study with maximum packet size, minimum reject, and spatial filtering. The gain was set to the level just below that at which colour flooding occurred. Mitral and tricuspid regurgitant jets were measured by planimetry in the view that gave the largest jet area, and the results were expressed as a percentage of the total right or left atrial area. ${ }^{2021}$

\section{STATISTICAL ANALYSIS}

All results are reported as mean (SD). Nonparametric data arising from subjective questionnaires, visual analogue scales, and the clinical assessment score were compared by the Wilcoxon signed rank test while parametric data arising from objective tests were compared by Student's paired $t$ test. Student's unpaired $t$ tests were used for inter-patient subgroup comparisons. If more than two comparisons were undertaken of a given variable the Bonferroni correction was used. A p value of less than 0.05 was regarded as significant.

\section{Results}

SUBJECTIVE ASSESSMENT

Figure 1 shows the patients' perception of "general well-being" and exercise capacity. General well-being was significantly better in

Figure 1 Perceived general well-being and exercise capacity in the study pacing modes (see text for details).

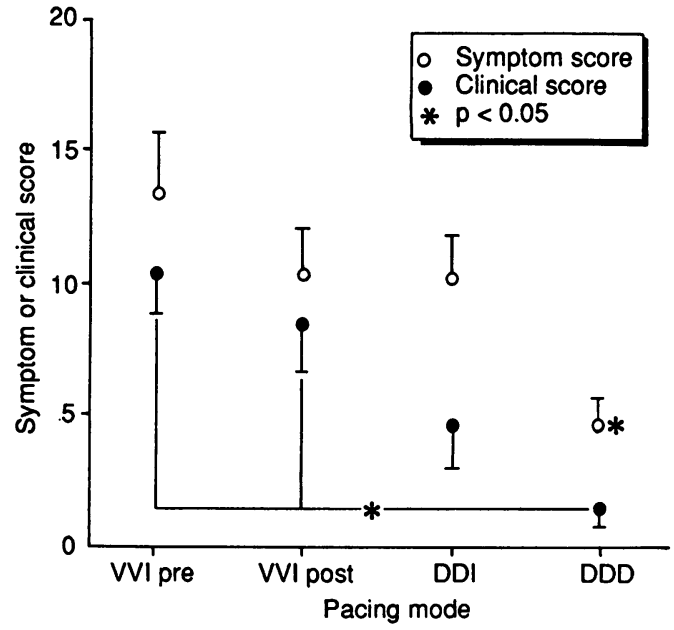

Figure 2 Symptom prevalence score and clinical assessment score in the four study pacing modes.

DDD mode than in either VVI pre mode or VVI post mode, $(p<0.01)$. Perceived exercise capacity was similar in VVI pre, VVI post, and DDI modes but was significantly greater in DDD mode $(\mathrm{p}<0.01)$.

There was no significant difference in perceived functional status in any mode throughout the study. However, specific symptom scores were significantly lower (that is, fewer symptoms) in DDD mode than in the remaining study modes ( $p<0.05$, fig 2 ).

Figure 3 shows the overall preferred and least acceptable modes which were selected after the end of the study. There was good correlation with the preferences indicated in the subjective questionnaires. VVI was not selected as an outright preferred mode by any patient. Sixty nine per cent selected DDD, one patient selected DDD or VVI pre $(6 \%), 12 \%$ DDI (one of the two patients had paroxysmal supraventricular arrhythmias), and $12 \%$ had no preference. No patient selected DDD as their least acceptable mode. However, two patients $(12 \%)$ found the DDI mode least acceptable. Both these patients had been randomised initially to the VVI post mode and then to DDD before being programmed to the DDI mode. The VVI pre mode was the least acceptable to three patients $(18 \%)$ while the VVI post mode was least acceptable to $50 \%$ of patients. The two patients who had no preference for any mode were not pacemaker dependent.

Three patients demanded early crossover to the next programmed mode during the study. One patient initially randomised to DDD mode after the pacemaker was upgraded could not tolerate either DDI or VVI modes thereafter. The remaining two patients had been randomised to DDD mode before VVI mode and found the latter intolerable. (One of these patients did not request early reprogramming from the DDI mode which followed VVI mode).

CLINICAL EXAMINATION

The clinical assessment score was significantly greater (that is, there were more signs of pacemaker syndrome) in both VVI modes than 


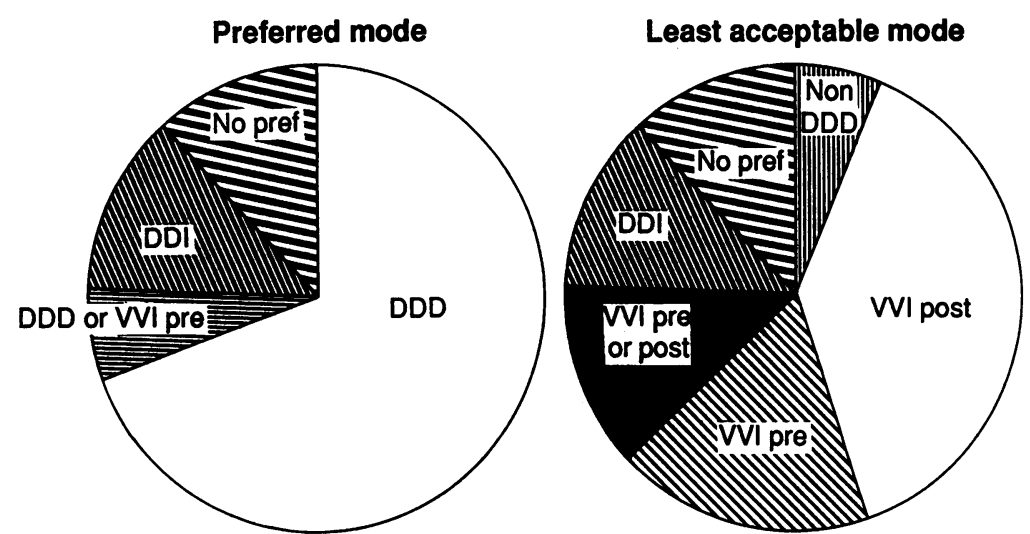

Figure 3 Preferred and least acceptable pacing modes defined by patients after completion of the study.

Figure 4 Objective study indices: exercise time while undertaking the CAEP exercise treadmill test and during staircase ascent (see text for details).

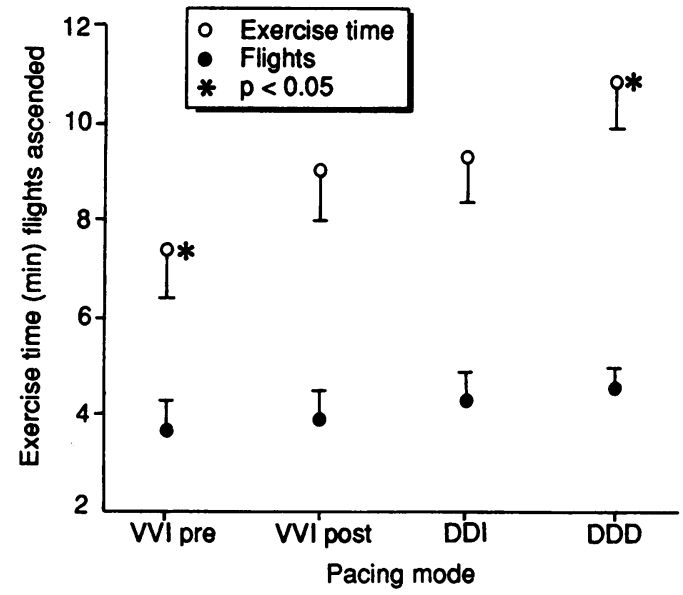

in the DDD mode ( $p<0.05)$, but did not differ from that in the DDI mode (fig 2). Mean arterial blood pressure measured at rest during clinical assessment did not significantly differ in any mode (VVI pre $103.6(4.0) \mathrm{mm} \mathrm{Hg}$, VVI post 103.2 (3.0) mm Hg; DDI 107.9 (3.4) $\mathrm{mm} \mathrm{Hg}$; DDD $107 \cdot 3$ (3.0) $\mathrm{mm} \mathrm{Hg}$ ).

OBJECTIVE ASSESSMENT

(i) Graded exercise treadmill tests (fig 4)

Exercise times were longer in dual modes, but

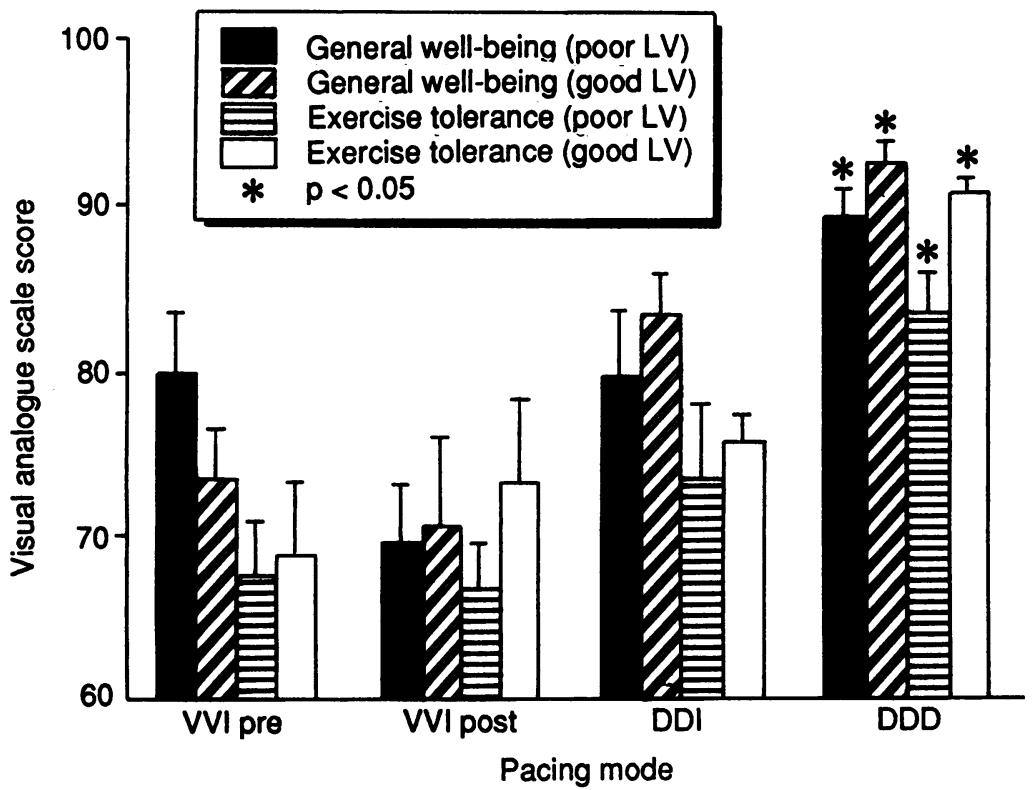

Figure 5 Perception of general well-being ( $G W$ ) and exercise capability (ET) according to left ventricular systolic function. Poor $L V$, left ventricular shortening $\leqslant$ $25 \%$; good $L V$, left ventricular shortening $>25 \%$. there were no significant differences between any modes except VVI pre mode and DDD $(7 \cdot 4$ $(1.0) \mathrm{min}$ and $10.9(1.0) \mathrm{min}$ respectively, $\mathrm{p}<0.05)$ during the CAEP protocol.

(ii) Staircase ascent (fig 4)

The mean number of stairs ascended was greater in dual modes than in the VVI pre or VVI post modes, but this difference did not reach statistical significance $(3 \cdot 7(0 \cdot 4)$ flights, in VVI pre mode; $3.9(0.4)$ flights in VVI post; 4.3 (0.4) flights in DDI mode, and $4 \cdot 6(0 \cdot 2)$ flights in DDD mode). The percentage change in heart rate during both of the above protocols was significantly greater in DDD than in the remaining modes, which did not significantly differ (CAEP treadmill test expressed as a percentage change in heart rate from rest to peak exercise in VVI pre $10.7(6.7)$, VVI post 16.6 (9.5); DDI 14.9 (6.0) ( $\mathrm{p}=\mathrm{NS}$ ), DDD mode $59.6(7.8)(p=0.001)$; staircase ascent expressed as percentage change in heart rate from rest to peak rate in VVI pre $12(6.3)$, VVI post $10.9(5.7)$; DDI $14.6(6.8)(\mathrm{p}=\mathrm{NS})$, DDD 46.5 (7.7) (p < 0.001).

Patient data were divided into two subgroups according to left ventricular systolic function assessed by echocardiography. ("Good LV" indicated fractional shortening greater than $25 \%$ and "poor LV" fractional shortening $25 \%$ or less (table 1 )).

Left ventricular function had no influence on the significant effects shown in the different modes on any variables discussed above (fig 5) except for exercise treadmill testing when the previously detected significant increase in exercise tolerance in DDD mode is no longer evident in either the "good" or "poor" left ventricle groups $(p=0.09$ and $p=0.065$ respectively with Bonferroni correction).

RADIOLOGICAL ASSESSMENT

There was no significant difference in the cardiothoracic ratio at any stage of the study; (VVI pre, $49.5(1 \cdot 1) \%$; VVI post, $51 \cdot 2(1.3) \%$; dual, $50 \cdot 75(1 \cdot 4) \%(p=N S))$. The incidence of pulmonary oedema was similar in all stages of the study (18\% in VVI pre and $24 \%$ in both VVI post and dual. The arbitrary pulmonary oedema severity scores in these patients were $1.7(0.6), 1.5(0.6)$, and $1.0(0.3)$, respectively $(\mathrm{p}=\mathrm{NS})$.

ECHOCARDIOGRAPHIC ASSESSMENT

Cardiac dimensions did not differ in any mode during the study. Left ventricular fractional shortening was similar in all modes (VVI pre, $27.9(2 \cdot 8) \%$; VVI post, $27 \cdot 1$ (2.4)\%; DDI, 26.7 (2.8)\%; and DDD, $25.5(2.5) \%(p=N S))$. Pulmonary arterial pressure was evaluable in all modes in only eight patients $(50 \%)$. In this cohort there was no significant difference in pulmonary artery pressure in any mode.

The incidence of mitral and tricuspid regurgitation was less in the DDD mode but this did not reach statistical significance (Mitral regurgitation: VVI pre, $50 \%$; VVI post, $63 \%$; DDI, $63 \%$; and DDD, $30 \%$. Tricuspid regurgitation: VVI pre, $63 \%$; VVI post, $63 \%$; DDI, $63 \%$ and DDD, 50\%). However, the extent of the regurgitant jets assessed by colour 
Figure 6 Extent of mitral and tricuspid regurgitation detected by colour flow Doppler echocardiography in the study pacing modes.

Figure 7 Cardiac output in each study pacing mode categorised according to patients' left ventricular systolic function.
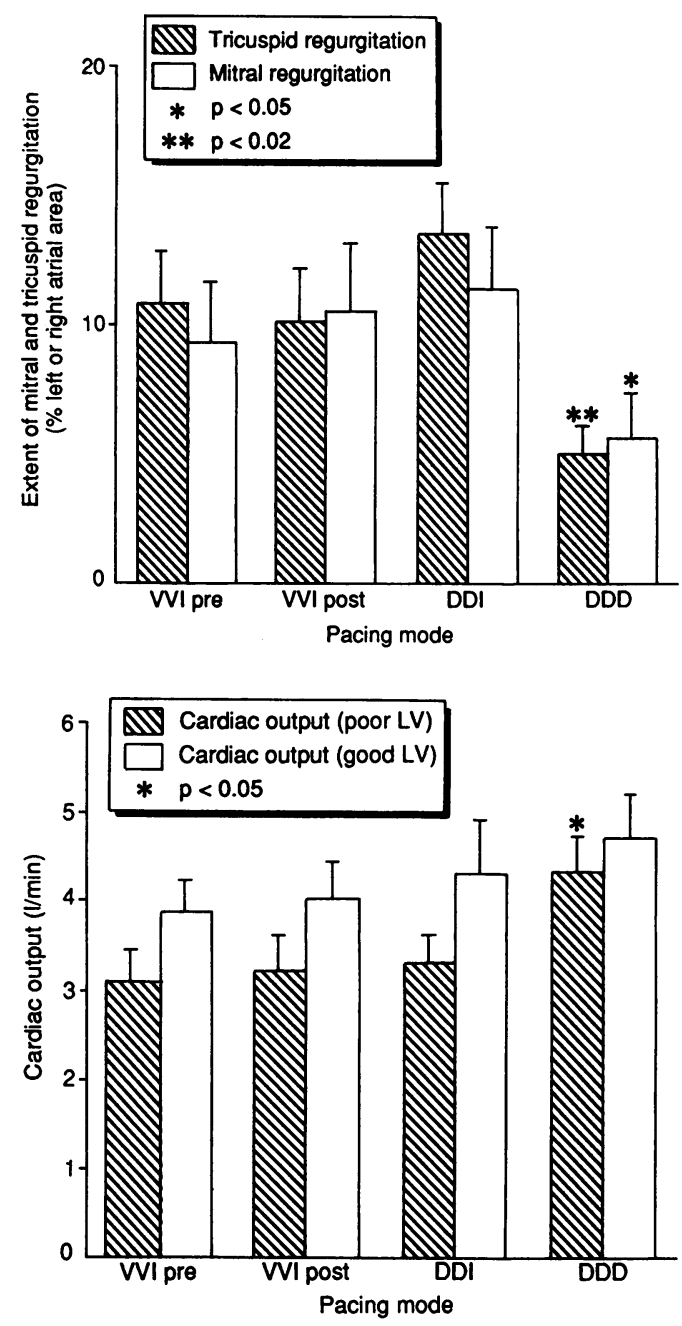

flow Doppler was significantly less in the DDD mode than in the remaining modes (fig 6).

Cardiac output was least in the single chamber modes and greatest in the DDD mode but these values were not statistically significant (cardiac output in VVI pre, $3.6(0.3) 1 / \mathrm{min}$, in VVI post, $3.6(0.4) 1 / \mathrm{min}$, in DDI $3.8(0.4)$ $1 / \mathrm{min}$; in DDD mode $4.5(0.3) 1 / \mathrm{min}$ $(p=0.065)$.

Echocardiographic data reanalysed with patients sub-divided according to left ventricular function showed no differences from combined data with the sole exception of cardiac output. In the subgroup with poor left ventricular function cardiac output was significantly greater in DDD mode $(4 \cdot 3(0.4) 1 / \mathrm{min}$ than in the remaining modes (VVI pre, $3.1(0.4)$ $1 / \mathrm{min}$, VVI post $3.2(0.4) 1 / \mathrm{min}$, DDI $3.3(0.3)$ $1 / \min (p=0.03))$. This was not evident in the subgroup with good left ventricular function (cardiac output in DDD mode, $4.7(0.3) 1 / \mathrm{min}$; VVI pre, 3.9 (0.25) $1 / \mathrm{min}$; VVI post, $4.0(0.3) \mathrm{l} /$ min; DDI, $4.3(0.4) 1 / \mathrm{min}$ ( $\mathrm{p}=\mathrm{NS})$, fig 7 ).

\section{Discussion}

The advantages of physiological, atrioventricular sequential pacing are now widely accepted with several studies showing improvement in objective and subjective indices in dual modes compared with VVI mode. ${ }^{42-24}$ In addition, patient characteristics do not allow identification of those at risk from the pacemaker syndrome before pacemaker implantation. It occurs at any age, in both sexes, as often in patients with morphologically normal hearts as in those with cardiac disease, with or without ventriculoatrial retrograde conduction, and it may occur at any time during ventricular demand pacing. ${ }^{1232526}$ Moreover VVI pacing, especially for sinus node disease, may adversely affect long-term prognosis. $^{97}$ Nevertheless VVI pacemakers are the most commonly used devices for all indications for permanent pacing worldwide. ${ }^{27}$

\section{MODE PREFERENCE}

This study provides evidence that when patients without the overt symptoms of the pacemaker syndrome (most VVI paced patients) are upgraded to dual chamber synchronous (DDD) pacing they show a considerable improvement in subjective and objective indices.

Mitsuoka et al found similar subjective benefit in patients paced with dual chamber devices for sick sinus syndrome, but many of these patients had retrograde ventriculoatrial conduction and overt signs of the pacemaker syndrome when paced in the VVI mode. ${ }^{28}$

These findings may result from the effects of the rate response offered by DDD mode but lacking in the remaining study modes except in the non-pacemaker dependent patients. However, subjective indices were worse in the VVIR mode than in the DDDR mode in patients with chronotropic incompetence-so that it seems that atrioventricular synchrony is a major factor resulting in improved patient acceptability. ${ }^{29}$

In the present study, the choice of least acceptable mode was highly dependent upon the programming sequence. Hence if VVI post was the first postoperative mode programmed, subjective scores were better than if the VVI post mode followed the DDD mode. The same was true for DDI and could explain why it was selected as the least acceptable mode by two patients and caused a request for early reprogramming in one case. (In both patients the VVI post mode was programmed before the DDD mode and the DDI mode was programmed after the DDD mode. Mode sequence had little effect on objective study indices, however. The DDI mode was little different from the VVI mode in most patients. This may be due to the lack of rate response in either mode although increased rate responsive pacing is required for less than $15 \%$ of the day during out of hospital activity. ${ }^{30}$ Because most of the day is spent at low heart rates, when dual chamber synchronous pacing occurs in DDI mode (with the "atrial kick" exerting its most beneficial effects ${ }^{31}$ ) in theory this mode should be haemodynamically better than the VVI mode and hence more acceptable to the patient. The most prevalent symptom in the DDI mode was "palpitations" (rather than "tiredness" in the VVI mode) and this may have been caused by loss of atrial synchrony on mild exertion as the atrial rate increased and the pacemaker, in effect, defaulted to VVI pacing. This persistent "mode switching" may be the feature that is 
least acceptable to most patients, and thus the DDI mode should be used only in selected patients with paroxysmal arrhythmias. ${ }^{32}$

There was a small, non-significant increase in perceived exercise capacity and a decrease in symptom prevalence in the VVI post mode compared with the VVI pre mode. This is likely to be a placebo response resulting from the implantation of a more sophisticated device.

OBJECTIVE STUDY INDICES

Nishimura and colleagues showed considerable falls in mean arterial blood pressure in patients with pacemaker syndrome that resolved with dual chamber pacing. ${ }^{3}$ We did not show any significant differences in any mode, probably because none of our patients had overt pacemaker syndrome.

Treadmill tests, but not staircase ascent, showed a significant increase in exercise tolerance in DDD mode compared with the VVI pre mode as has previously been shown. ${ }^{43}$ However, the staircase ascent did not exercise all patients to their maximum tolerance and thus differences were less well demarcated during this "everyday activity" stress protocol.

Exercise tolerance was greater in the VVI post mode than in the VVI pre mode (although not significantly so), partly because of a placebo response (most subjects might have expected some improvement in exercise capability after the upgrading). There might also be a training effect between these modes, despite familiarisation with the treadmill before study induction and the randomisation of postoperative pacing modes, as all patients necessarily performed at least one more treadmill protocol in the VVI post mode than in the VVI pre mode.

Reanalysis of data according to echocardiographically derived left ventricular fractional shortening and not the ejection fraction was based on the findings of Gomes et al who showed that the asymmetry of ventricular depolarisation induced by right ventricular apical pacing causes significant errors in the estimation of ejection fraction. ${ }^{33}$

Stewart et al using similar techniques showed that the increase in cardiac output between VVI and DDD modes was greatest in patients with the most overt symptoms of the pacemaker syndrome when paced in VVI mode. ${ }^{34}$ Interestingly, although there was an increased cardiac output detected in the DDD mode compared with the remaining modes this did not reach statistical significance in our study population. Thus it seems that patients who tolerate VVI pacing well have a less considerable haemodynamic benefit from dual chamber pacing at rest despite clear subjective improvement during everyday activity.

In the subgroup with poor left ventricular function, however, there was a significant increase in cardiac output at rest confirming the findings of Pearson et al. ${ }^{35}$ Although these patients showed more haemodynamic benefit in terms of cardiac output, subjective scores were similarly favourable to DDD mode in both the subgroups. This suggests that the improved symptoms and general health perception is largely independent of left ventricular systolic function. Similar findings with rate responsive pacing modes confirm that improved cardiac output is not the sole factor in patient preference for atrioventricular sequential pacing. ${ }^{29}$

Both echocardiography and chest radiology confirmed that the cardiac dimensions did not vary during long-term pacing in any study mode. This is contrary to findings in patients with overt pacemaker syndrome, where heart failure on chest radiography (and clinically) was more often seen in the VVI than in the DDD mode. ${ }^{122}$

Although the incidence of both mitral and tricuspid regurgitation was similar in al modes, the extent of both mitral and tricuspid regurgitation was significantly less in DDD mode and was independent of left ventricular function. Valve incompetence has been proposed as a cause of the pacemaker syndrome,$^{36}$ and the extent of valve regurgitation, but not the incidence, may be a factor in the genesis of "subclinical" pacemaker syndrome.

Only four of 14 patients assessed in the present study had consistent retrograde ventriculoatrial conduction and the small size of this subgroup precludes meaningful statistical analysis. Rediker et al have recently identified patients with an increase in systolic blood pressure from VVI to DDD pacing and with ventriculoatrial conduction who benefited most from dual chamber pacing. ${ }^{37}$ These findings suggest that our study population was free from the pacemaker syndrome and unlikely to benefit from DDD pacing. Despite this, most patients preferred the DDD mode and found the VVI mode least acceptable after completion of the study. Retrospective analysis of data to predict maximum benefit from dual chamber pacing in symptom free VVI paced patients was inappropriate because of disparate sample sizes. Larger patient numbers might allow such analysis.

\section{CLINICAL IMPLICATIONS}

Few studies have compared VVI and DDD with DDI mode pacing. Although patient numbers in this study were small, with each acting as their own control, the results suggest that DDI mode is subjectively and objectively similar to the VVI mode and not as good as the DDD mode. The DDI mode should therefore be used only in patients who cannot tolerate DDD pacing (because of frequent paroxysmal atrial arrhythmias).

Our data suggest that "subclinical" pacemaker syndrome occurs in nearly three quarters of symptom free VVI paced patients. Combined with the incidence of overt pacemaker syndrome in up to $20 \%$ of patients with VVI devices, ${ }^{1} 80 \%$ of all VVI paced patients without atrial fibrillation would benefit from DDD pacing, and this effect persists even after long-term VVI mode pacing.

Comparable findings were seen in the VVIR mode when it was compared with dual chamber rate responsive modes, ${ }^{29}$ which suggests that a similar "subclinical" pacemaker syndrome may exist with this pacing mode. (It is well 
known that VVIR mode can cause severe pacemaker syndrome..$^{38}$ )

Hence, VVI pacing should be used only in patients with chronic or persistent atrial fibrillation with good chronotropic response to exercise, or with transient bradycardic arrhythmias. This investigation confirms the adage: If there is a $P$ wave available, sense it and synchronously pace the ventricle if necessary.

We thank Miss Lynne Chappell for typing the paper.

1 Ausubel K, Furman S. The pacemaker syndrome. Ann Intern Med 1985;103:420-9.

2 Alicandri C, Fouad FM, Tarazi RC, Castle L, Morant V. Three cases of hypotension and syncope with ventricular pacing: possible role of atrial reflexes. $\mathrm{Am} J$ Cardio 1978;42:137-42.

3 Nishimura RA, Gersh BJ, Vlietstra RE, et al. Hemodynamic and symptomatic consequences of ventricular pacing. a ACE 1982;5:903-10.

4 Perrins EJ, Morley CA, Chan SL, Sutton R. Randomised controlled trial of physiological and ventricular pacing. Controlled trial of physiologit $J$ 1983;50:112-7.

5 Nishimura RA, Gersh BJ, Holmes DR, et al. Outcome of dual chamber pacing for the pacemaker syndrome. Mayo

6 Torresani J, Bogosti A, Allard-Latour P, et al. Pacemaker syndrome with DDD pacing. $P A C E$ 1984;7:1148-51.

7 Cunningham TM. Pacemaker syndrome due to retrograde conduction in a DDI pacemaker. Am Heart $J$ 1988; 115:478-9.

8 Barold SS, Mugica J, eds. The third decade of cardiac pacing advances in technology and clinical applications. New York: Futura Publishing Co, 1982:22-42.

9 Rosenqvist $M$. Atrial pacing for sick sinus syndrome. Clin Cardiol 1990:13:43-7.

10 Camm AJ, Butrous GS. Clinical cardiac electrophysiology. In: Julian DG, Camm AJ, Fox KM, et al, eds. Diseases of the heart. London: Balliere Tindall, 1989:233-53.

11 Price DD, McGrath PA, Rafii A, Buckingham B. The validation of visual analog scales as ratio scale measures for validation of visual analog scales as ratio scale measures
chronic and experimental pain. Pain 1983;17:45-56.

12 Von Olshausen KE, for the German and Austrian xamoterol on Olshausen KE, for the German and Austrian xamoterol study group. Double-blind placebo-controlled comparison of digoxin \&

13 Goldman L, Hashimoto B, Cook F, Loscalzo A. Comparative reproducibility and validity of systems for ss: advantages of new specific activities scale. Circulation 1981;64:1227-34

14 Wilkoff BL, Corey J, Blackburn G. A mathematical model of the cardiac chronotropic response to exercise. J Electrophysiol 1989;3:176-80.

15 Sahn D, DeMaria AN, Kisslo J, Wetman A. Recommendations regarding quantitation in $M$ mode echocardiography: results of a survey of echocardiographic graphy: results of a survey of echocar

16 Magnin PA, Stewart JA, Myers S, et al. Combined Doppler and phased-array echocardiography estimation of cardiac and phased-array echocardiography

17 Chandraratna PA, Nanna M, McKay C, et al. Determination of cardiac output by transcutaneous continuous wave ultrasonic Doppler computer. Am J Cardiol 1984;53: 234-57.
18 Haites NE, McLennan FM, Mowat DHR, Rawles JM Assessment of cardiac output by the Doppler ultrasound technique alone. Br Heart J 1985;53:123-9.

19 Berger M, Haimowitz A, Van Tosh A, et al. Quantitative assessment of pulmonary hypertension in patients with tricuspid regurgitation using continuous wave Doppler ultrasound. J Am Coll Cardiol 1985;6:359-65.

20 Chopra HK, Nanda NC, Fan P, et al. Can two dimensional echocardiography and Doppler color flow mapping identify the need for tricuspid valve repair? $\mathrm{J}$ Am Coll identify the need for tricus

21 Helmcke F, Nanda NC, Hsiung MC, et al. Color Doppler assessment of mitral regurgitation with orthogonal planes. assessment of mitral regurgi

22 Kruse IB, Arnman K, Conradson TB, Ryden L. A comparison of the acute and long-term effects of ventricular inhibited and atrial synchronous ventricula inhibited pacing. Circulation 1982;65:846-55.

23 Kristensson B-E, Arnman K, Ryden L. The haemodynamic importance of atrioventricular synchrony and rate increase at rest and during exercise. Eur Heart 1985;6:773-81.

24 Haas JM, Strait GB. Pacemaker-induced cardiovascular failure. Hemodynamic and angiographic observations. Am J Cardiol 1974;33:295-9.

25 Furman S. Cardiac pacing and pacemakers. VI. Analysis of pacemaker malfunction. Am Heart J 1977;94:378-86.

26 Rosenqvist M, Brandt J, Schuller $H$. Long-term pacing in sinus node disease: effects of stimulation mode on sinus node disease: effects of stimulation mode on 1988;116:16-22.

27 Tyers FO. Current status of sensor modulated rate adaptive cardiac pacing. J Am Coll Cardiol 1990;15:412-8.

28 Mitsuoka T, Kenny RA, Yeung TA, Chan SL, Perrins JE Sutton $R$. Benefits of dual chamber pacing in sick sinus syndrome. Br Heart $J$ 1988;60:338-47.

29 Sulke AN, Chambers JB, Dritsas A, Sowton E. A randomised double blind crossover study of four rate responsive pacing modes. J Am Coll Cardiol 1991;17: 696-706.

30 Sulke AN, Pipilis A, Bucknall CA, Sowton E. Quantitative analysis of contribution of rate response in three different VVIR pacemakers during out of hospital activity. $P A C E$ 1990;13:37-44.

31 Karlof I. Hemodynamic effects of atrial triggered versus fixed rate pacing at rest and during exercise in complete heart rate pacing at rest and during exercise in con
block. Acta Med Scand 1975;197:195-206.

32 Bana G, Locatelli V, Piatti L, et al. DDI pacing in bradycardia-tachycardia syndrome. PACE 1990;13. $264-70$.

33 Gomes JAC, Damato AN, Akhtar $M$, et al. Ventricular septal motion and left ventricular dimensions during abnormal ventricular activation. Am J Cardiol 1977; 39:641-5.

34 Stewart WJ, Dicola VC, Harthorne JW, et al. Dopple ultrasound measurement of cardiac output in patients with physiologic pacemakers. Am J Cardiol 1984;54:308-12.

5 Pearson AC, Janosik DL, Redd RM, et al. Hemodynamic benefit of atrioventricular synchrony: predication from baseline Doppler echocardiographic variables. J Am Coll Cardiol 1989;13:1613-21.

36 Naito M, Dreifus LS, Mardelli TJ, et al. Echocardiographic features of atrioventricular and ventriculoatrial conduction Am J Cardiol 1980;46:625-33.

37 Rediker DE, Eagle KA, Homma S, et al. Clinical and hemodynamic comparison of VVI versus DDD pacing in patients with DDD pacemakers. Am J Cardiol 1988; patients with

38 Liebert HP, O'Donoghue S, Tullner WF, et al. Pacemake syndrome in activity responsive VVIR pacing. $\mathrm{Am}$ Cardiol 1989;64:124-6. 